\title{
Crónica de las Jornadas ¿Hacia dónde va el Estado de las Autonomías? Córdoba, 18-21 de julio de 2005*
}

\author{
Antonio M믈 Bueno Armijo** \\ Ciro Milione**** \\ Becarios de Investigación \\ Departamento Derecho Público y Económico \\ Universidad de Córdoba
}

I. En el marco de los Cursos de Verano de la Universidad de Córdoba, Corduba, se celebraron durante los pasados días 18, 19, 20 y 21 de julio de 2005 las Jornadas ¿Hacia dónde va el Estado de las Autonomías?

Con estas Jornadas, organizadas por el Centro de Estudios Andaluces en colaboración con la Universidad de Córdoba, se pretendía satisfacer la demanda de aportaciones críticas, elaboradas en la Academia, sobre el actual proceso de reformas estatutarias, según expuso el Coordinador de las Jornadas, el Prof. Titular de Derecho Constitucional de la Universidad de Córdoba e Investigador responsable del Área Jurídico-Política del Centro de Estudios Andaluces, Dr. D. Miguel J. Agudo Zamora. No dudó, en este sentido, el Prof. Agudo, en congratularse por poder contar en un mismo foro universitario con Catedráticos de Derecho Constitucional de todas las Universidades Andaluzas.

También el Prof. Alfonso Yerga, Director-Gerente del Centro de Estudios Andaluces, remarcó en la presentación de las Jornadas la señalada oportunidad de las mismas, una vez presentado el informe de la ponencia del Parlamento andaluz para la reforma del Estatuto de Autonomía para Andalucía y

\footnotetext{
*Estas crónicas han sido elaboradas gracias a la colaboración del Centro de Estudios Andaluces.

**SEJ 2004-03791. "El papel de las Administraciones públicas y sus distintos instrumentos de actuación en la economía y la sociedad”.

***SEJ-372. "Democracia. Pluralismo. Ciudadanía".
} 
muy avanzados los trabajos de reforma estatutaria en las Comunidades Autónomas de Cataluña y Valencia.

Tras este breve acto de presentación, el propio Prof. Agudo explicó la estructura de las Jornadas, indicando que el orden de las conferencias suponía una progresión desde lo más general y teórico hasta lo más concreto y cercano. De este modo, a las primeras conferencias, de marcado carácter dogmático y doctrinal (concepto de Nación, modelos de distribución territorial de poder) habrían de suceder otras de ámbito temático más acotado (reforma de los Estatutos de Autonomía, desde una perspectiva genérica y comparada) para acabar en la exposición y el análisis de los problemas, más cercanos, de la reforma del Estatuto de Autonomía de Andalucía (EAA).

II. Conforme al plan trazado, la primera jornada contó con las ponencias de los dos Catedráticos "de la casa", los Profs. Drs. de la Universidad de Córdoba D. José Acosta Sánchez y D. Pablo Lucas Murillo de la Cueva, a cuyas intervenciones siguió un animado coloquio sobre los temas expuestos por ambos, con el que se cerró esta primera sesión de trabajo.

A) La primera de las ponencias, titulada "El modelo de distribución territorial del poder diseñado por la Constitución de 1978" y a cargo del Prof. Lucas Murillo de la Cueva, tuvo como objeto la exposición y el análisis de la evolución del Estado Autonómico. El Prof. Lucas empezó destacando cómo la ordenación del Estado Constitucional se configuró, en un principio, sobre los patrones centralizadores de la tradición administrativa revolucionario-napoleónica y como ésta no experimentó variaciones sustanciales hasta la entrada en vigor del texto constitucional vigente. Este modelo se vería, además, confirmado durante el periodo de la dictadura franquista, en el que la reacción centralizadora y uniformista llevó a eliminar todo vestigio de autonomía territorial y a perseguir con particular virulencia cualquier forma de pluralismo ajena a los estrechos horizontes ideológicos del régimen. Posteriormente, los programas electorales de las elecciones del 15 de junio de 1977 y la composición de las Cortes elegidas sellaron definitivamente la vinculación entre libertad y autonomía sobre la que descansa hoy el pacto constitucional.

Como recordó el ponente, estos dos objetivos, libertad y autonomía, originaron serias dificultades a la hora de su plasmación en formulaciones jurídico-positivas concretas. Lo acreditaría la misma génesis del art. 2 del texto fundamental y la posterior elaboración de su título VIII. Entre otras, las principales dificultades fueron, a juicio del Prof. Lucas, la originada por la introducción en el art. 2 CE del término "nacionalidades", y, junto a ella, los espa- 
cios que el título VIII dejaba abiertos a desarrollos futuros, generando dudas de entidad y notables preocupaciones. En este sentido, el Prof. Lucas consideró interesante reseñar que si, en 1978, el término nacionalidades suscitó una notable perplejidad, en la actualidad el acento se pone sobre el entendimiento que haya de darse al concepto "nación española".

Naturalmente, la innovación realizada por el art. 2 CE planteó, en su momento, un intenso debate encaminado a cuestionar, desde el punto de vista de la Teoría del Estado, la posibilidad de coexistencia entre "nación" y "nacionalidades", pues se consideraba que cada una excluía a la otra. Con el transcurso del tiempo este debate fue pacificándose, sobre todo por el gradual pero rápido proceso de transferencia de competencias, pero el problema ha pervivido y lo cierto es que han sido el Partido Nacionalista Vasco y la coalición gobernante en Cataluña quienes han avivado esa polémica. En ambos supuestos se ha insistido en el "hecho diferencial" y en la consiguiente necesidad de que esas CCAA disfruten de un régimen de autogobierno distinto -más amplio- al de las otras Comunidades.

Según el Prof. Lucas, hoy en día parece que se ha llegado a una inversión del prisma desde el que se observa el mismo problema: si al principio la defensa de una concepción unitaria y centralizadora estimaba incompatible la existencia de nacionalidades en el seno de la Nación, ahora ocurre que, al margen de las consistentes salvaguardias jurídicas de la unidad estatal, no se pretende seriamente cuestionar la existencia de estas nacionalidades. Más bien, a lo que parece apuntarse es a la búsqueda de nuevos equilibrios en la distribución territorial del poder. Para ello, existirían instrumentos constitucionales aptos para alterar dicha distribución del poder, de los cuales los más llamativos, aunque no los únicos, son los contemplados en el art. $150 \mathrm{CE}$.

B) Por su parte, en la segunda ponencia del día, "Problemática de la Nación y futuro del Estado Autonómico”, el Prof. Acosta Sánchez regaló a los oyentes un extraordinario estudio sobre el concepto de Nación. Su exposición arrancó con una prospección histórica en busca de los orígenes de esta construcción, remontándose hasta el "Pacto del Pueblo" inglés de 1648, resultado de la revolución de 1642. Es en este contexto original donde se desvela el contenido y la significación del concepto "Nación". En efecto, el fin de la monarquía y la deposición del rey Carlos I en la revolución de 1642, supuso la eliminación del sujeto político que ostentaba el poder supremo de la sociedad inglesa -la soberanía, en sentido estricto-, y que, por ello mismo, se erigía en principal elemento de cohesión de dicha sociedad. Los revolucionarios ingle- 
ses, ante la necesidad de llenar el vacío que dejaba la eliminada monarquía, se habrían visto compelidos a buscar un nuevo sujeto político capaz de erigirse en titular de la soberanía. El resultado, históricamente inédito y cuya principal novedad radicó en que, por primera vez, el soberano era un sujeto abstracto, fue el concepto de Nación.

El éxito del nuevo sujeto soberano, capaz de mantener la cohesión de la sociedad y de servir de base a la construcción del Estado, quedo refrendado al ser posteriormente utilizado por los revolucionarios franceses (art. 3 de la Declaración de Derechos del Hombre y del Ciudadano) y por los constituyentes españoles de 1812 (art. 3 de la Constitución gaditana).

A juicio del Prof. Acosta, el triunfo de la Nación sobre el Monarca en España contó a lo largo de todo el s. XIX con el apoyo de las fuerzas revolucionarias y progresistas. En consecuencia, y por paradójico que pudiera parecer hoy día, ideólogos federalistas como Pi i Margall también reconocieron y defendieron, expresamente, la existencia de una única Nación española. De modo paralelo, el federalismo norteamericano, alemán e italiano obedecerían, en última instancia, al reconocimiento de una única Nación, sostén y justificación de sus respectivos Estados.

No obstante, y una vez vencido el monarca, los movimientos sociales de finales del s. XIX y principios del XX, en su reacción frente a las democracias burguesas, habrían de presentar un nuevo candidato a la titularidad de la soberanía: el Pueblo. La tensión entre soberanía nacional y soberanía popular puede rastrearse, así, en la Constitución Española de 1931 que, al constituir España como "República de trabajadores", no hace pronunciamiento expreso alguno sobre el titular de la soberanía. Esta tensión, de hecho, se habría vuelto a reproducir casi cincuenta años después en la Constitución Española de 1978, resolviéndose en el art. 1.2 al indicar, mediante una fórmula de consenso, que "la soberanía nacional reside en el pueblo español del que emanan los poderes del Estado".

Sin embargo, es la propia CE, en su art. 2 en conexión con el art. 151 y con la Disposición Transitoria $2^{a}$, la que introduce unos novedosos sujetos políticos, las Nacionalidades. Éste nuevo concepto, desconocido en nuestra historia constitucional y en el Derecho comparado, supondría, según el Prof. Acosta, una nueva forma de organización del Poder, irreductible a la mera organización territorial (descentralización política) propuesta en el título VIII CE. Esto es, las Nacionalidades exigirían un verdadero poder originario, lo que implicaría su configuración como nuevos rivales de la Nación en la liza por alzarse con la soberanía, único poder originario posible. 
Como consecuencia de todo lo anterior, se descubre que las actuales reivindicaciones, desde posiciones nacionalistas, sobre el reconocimiento de alguna Comunidad Autónoma como Nación, se situarían en un proceso tendente a la construcción de un Estado confederal -no federal-, cuyo único modelo histórico parecería situarse en los Estados Confederados del Sur de los Estados Unidos durante la guerra de secesión.

III. A) La segunda jornada se abrió con la ponencia a cargo del Prof. Dr. D. Antonio Porras Nadales, Catedrático de Derecho Constitucional de la Universidad Pablo de Olavide, con el título "La reforma de los Estatutos de Autonomía”. El Prof. Antonio Porras empezó analizando las causas de esta demanda generalizada de reforma de los Estatutos de Autonomía, deteniéndose sobre algunas de ellas, como, por ejemplo, la simple constatación de que un sistema, una vez consolidado, precisa evolucionar.

El Prof. Porras ordenó dichas causas en tres categorías. En primer lugar, desde un punto de vista político, señaló cómo desde algunos sectores de la opinión pública se está percibiendo el proceso de reformas como una situación más próxima al chantaje emocional o al oportunismo político (amparado en la dependencia del Gobierno central de las minorías nacionalistas catalanas) que a un verdadero proceso reformador.

En segundo lugar, situando el proceso de reformas abierto en nuestro país en un contexto global y comparado, el Prof. Porras Nadales recordó que los fenómenos de "devolución" constituyen una forma de evolución general de las instituciones democráticas en el proceso de globalización del siglo XXI. Junto a ellos, el objetivo último del proceso de reforma sería una "segunda descentralización" hacia la autonomía local. En tercer lugar, y desde una perspectiva evolutiva interna, esta etapa de reformas parece ser consecuencia de un proceso histórico de aprendizaje institucional por parte de las CCAA, que plantea cuestiones de fundamental importancia, tales como si se han cumplido los objetivos prefijados, si ha sido operativo el sistema de competencias o, en definitiva, si ha funcionado el sistema institucional.

Fijados los términos en que se está desarrollando el proceso, el Prof. Porras Nadales se enfrentó a la cuestión relativa al objeto concreto de las reformas estatutarias, empleando para ello dos líneas de análisis diferenciadas.

La primera de estas líneas plantea el problema desde el punto de vista del debate político constitucional y de un posible nuevo reparto de poderes y de finanzas, trasladando la atención a una dimensión aparentemente "cuantitativa" 
del problema. Asistimos así a un fenómeno por el que el proceso autonómico, inexorablemente, se ha ido confundiendo con otro proceso, el de transferencias de competencias, a través del que las CCAA han venido recibiendo dosis crecientes de competencias y recursos. Sería, por lo tanto, ésta la causa de un singular "síndrome de abstinencia" que afectaría a las CCAA, obligadas a pedir siempre más, en términos de competencias y financiación, al gobierno central.

Según el Prof. Porras Nadales el problema consistiría no ya en consentir un traslado cada vez más importante de poderes a las CCAA, sino en determinar de una forma definitiva y cierta los términos de este reparto de competencias, a fin de evitar posibles abusos por parte del poder central. Evidentemente, hasta ahora no se ha sido capaz de delimitar ese reparto de competencias, objetivo difícilmente alcanzable en un contexto como el actual en que se asiste a un proceso de traspaso y desarrollo de normativas supranacionales.

La segunda línea de análisis consiste, en cambio, en un estudio conducido desde una dimensión técnico-jurídica que se traduce en un planteamiento cualitativo de la reforma: no se trataría de redefinir el reparto de poderes, sino de introducir modificaciones capaces de aportar mejoras al funcionamiento de todo el sistema de relaciones entre el Estado central y las CCAA. En este contexto, asistimos además a una demanda cada vez más fuerte dirigida a una ampliación del sistema de derechos, puesto que es indiscutible el fenómeno expansionista de éstos, tanto en el ámbito nacional (con la Constitución de 1978) como en el supranacional (y, en especial, europeo, con la Carta de Niza, la Constitución Europea, etc.).

A este respecto, el Prof. Porras Nadales consideró oportuno distinguir entre derechos de carácter político y derechos sociales, señalando cómo, por estar los primeros consagrados en la CE, parece imposible configurar nuevas situaciones jurídicas de esta tipología a nivel de Comunidades Autónomas, lo que causaría importantes discriminaciones. Sin embargo consideraciones distintas merecen los derechos sociales, para los que el Prof. Porras Nadales no consideró necesaria una nueva declaración sino, más bien, una acción dirigida a conseguir más eficacia a través de políticas públicas. En este sentido, las políticas públicas, determinando una concreta conexión entre competencias, derechos y valores, pueden considerarse como la principal innovación contemporánea del intervencionismo.

Con estas últimas consideraciones concluyó esta interesantísima ponencia a la que siguió un breve debate, con el que se aclararon las ideas anteriormente expuestas. 
B) La segunda ponencia del día 19 de julio, bajo el título "La reforma de los Estatutos de Autonomía de Cataluña, el País Vasco y Galicia”, fue realizada por el Prof. Dr. D. Santiago Roura, Profesor Titular de la Universidad de La Coruña, quien quiso empezar su intervención deteniéndose, él también, en los motivos y las causas del proceso de reforma de los Estatutos de Autonomía.

Se manifestó, así, claramente a favor de las reformas por varias razones, entre las cuales destacó la natural constatación de que han trascurrido ya 25 años desde la aprobación de la Constitución -coincidiendo con las opiniones del Prof. Porras Nadales-, y la necesidad de abordar la reforma tanto por motivos históricos como por motivos meramente evolutivos.

A pesar de eso, el Prof. Roura no se mostró completamente de acuerdo con la forma en la que se está desarrollando ese proceso. A su juicio, las reformas se estarían planteando casi exclusivamente en términos de incremento de las competencias y no de redefinición y aquilatamiento de las ya existentes. Junto a ello, la bipolarización del debate público actual, donde se estaría con España o en contra de ella, así como el estado de dependencia política del Gobierno central hacia las minorías nacionalistas habrían puesto en peligro el entero proceso de reforma.

El Prof. Roura analizó entonces los casos concretos de lo que él mismo definió como "un muerto, un enfermo y un nasciturus", refiriéndose, en este sentido, respectivamente a los proyectos de reforma de los estatutos vasco, catalán y gallego. Sobre el primero, el profesor de La Coruña se limitó a subrayar la necesidad de replantear por completo el Plan Ibarretxe, aunque auguró un futuro incierto por cuanto los intereses electorales hacen del Estatuto una cuestión poco llamada al consenso. Sobre el segundo, Roura advirtió que, una vez negado el apoyo del PP, el tripartito debía buscar apoyo de CiU, con los riesgos de inconstitucionalidad que sus exigencias parece que podrían conllevar. Por último, y en referencia al Estatuto gallego, el profesor afirmó no creer que el PSOE fuera a "pagar el peaje" de definir Galicia como nación tal y como propone su socio, el BNG. En cualquier caso, el Prof. Roura insistió en que el éxito de las reformas estatutarias no se lograría hasta que realmente se aborde una reforma constitucional que se adentre en el modelo federal y solidario de Estado.

En este sentido, criticó duramente el sistema de financiación que mantiene las ventajas fiscales del País Vasco y Navarra porque, en su opinión, la financiación de las autonomías debe responder a un modelo solidario y no discriminatorio. Aún más, la financiación no debería ser un tema de discusión en los estatutos autonómicos. 
En última instancia, y a pesar de tener una opinión claramente partidaria de la reforma de la Constitución, el Prof. Roura se mostró más realista al aceptar que en el contexto en el que nos encontramos lo mejor que podría pasar sería reeditar el acuerdo de la Transición y consensuar las reformas estatutarias, manteniendo la Constitución.

C) Concluyó esta segunda sesión situando el debate de las jornadas, finalmente, en la específica cuestión de la reforma del Estatuto de Autonomía de Andalucía (EAA). El encargado de introducirnos en la materia fue el Prof. Dr. D. José Luis García Ruiz, Catedrático de Derecho Constitucional de la Universidad de Cádiz, quien planteó en su conferencia los argumentos a favor y en contra de la necesidad real de reformar el EAA.

En primer lugar, comenzó destacando los desajustes, frecuentemente señalados por la doctrina, entre las previsiones que contenía el Estatuto andaluz y el modo en que se han desarrollado las instituciones andaluzas en la realidad. Por ejemplo, no existe referencia alguna en el texto estatutario a órganos como la Cámara de Cuentas o el Consejo Consultivo de Andalucía, ni se prevé tampoco entre los poderes del Presidente de la Junta de Andalucía el de disolver el Parlamento andaluz, situaciones éstas que han tenido que resolverse vía legislativa. Tampoco son desdeñables desajustes como la previsión de que la Administración periférica de la Junta de Andalucía se realizaría a través de las Diputaciones provinciales (art. 4.4 EAA) o el desfase de algunas preocupaciones sociales que impregnan el texto estatutario, como la situación de los emigrantes o la reforma agraria.

Consecuentemente, el Prof. García Ruiz abogó por la adecuación del Estatuto a la realidad sobrevenida como primera cuestión a abordar en la reforma estatutaria. Esta puesta al día se enmarcaría en una primera batería de modificaciones, a la que denominó "reformas bilaterales", que no afectarían al modelo constitucional en su conjunto, sino sólo a la Comunidad andaluza y a sus relaciones con el Estado central. En este mismo grupo se incluirían la eliminación de algún artículo transitorio del Estatuto (capitalidad, sede del TSJ), la inclusión o modificación de algunas cuestiones de Derecho parlamentario interno (sistema de elección de senadores autonómicos, elección del Presidente por el sistema de lista más votada), mejoras técnicas en la enunciación de competencias y, muy especialmente, la reforma del régimen local (descentralizacion, el papel de las entidades supramunicipales y financiación).

Fuera de este primer grupo quedarían otras posibles reformas, que irían más allá de las cuestiones meramente internas de la Comunidad Autónoma 
andaluza o de sus relaciones con el Estado central, afectando al propio modelo de Estado actualmente vigente. Estarían aquí comprendidos los posibles traspasos de competencias estatales (vía art. 150.2 CE) o la inclusión de nuevos sistemas de participación en la fase ascendente de creación del Derecho europeo comunitario (papel de los Parlamentos autonómicos, representación internacional de las CCAA, etc.).

Ahora bien, respecto a este segundo grupo de reformas ("reformas multilaterales") se haría necesaria la concurrencia de una nueva "convención constitucional". En efecto, cada una de las grandes etapas de la construcción del Estado autonómico se habría llevado a cabo previo pacto o acuerdo entre las principales fuerzas políticas, denominadas por el Prof. García Ruiz "convenciones constitucionales". Así ocurrió con la propia Constitución, con la solución al "problema andaluz", con los Pactos de la Moncloa de 1981 y el Pacto Autonómico de 1992 o, finalmente, con las reformas de los Estatutos navarro y gallego de 2001. En consecuencia, una reforma estatutaria, en Andalucía u otra Comunidad, que implicase avanzar hacia una nueva etapa del Estado autonómico, debería ir precedida de una nueva y correlativa "convención constitucional".

IV. Las conferencias de la tercera sesión de las jornadas, dictadas durante la mañana del miércoles 20 de julio, entraron ya en la exposición y debate de aspectos concretos del proceso andaluz de reforma estatutaria. En particular, se trató del encaje jurídico del EAA en el ordenamiento constitucional y comunitario, de los principios y derechos que informan su reforma, y del sistema de gobierno que en él se consagra.

A) Como ya había sido puesto de manifiesto en algunas intervenciones anteriores, una de las más preocupantes lagunas del Estado autonómico se encuentra en la falta de previsiones sobre el papel que las CCAA deban tener en la Unión Europea (UE). A este respecto, fue el Prof. Dr. D. Francisco Balaguer Callejón, Catedrático de Derecho Constitucional de la Universidad de Granada, el encargado de analizar la reforma del EAA en el marco del Estado autonómico y del proceso de constitucionalización de la UE.

El punto de partida de su ponencia radicó en la situación patológica que supone la ausencia de toda mención al Estado autonómico y a la UE en el texto de la CE. Dicha situación, históricamente justificada, no obsta a que en la realidad coexistan tres ordenamientos jurídicos diferenciados (comunitario, estatal, autonómico), cuyas relaciones se rigen por reglas no siempre claras. 
En este contexto, la reforma del EAA debería tener en cuenta, en primer lugar, el marco comunitario. A este respecto, resultaría oportuna la inclusión de referencias expresas al proceso de construcción europea en el texto del nuevo EAA, como parece que también se hará en el nuevo Estatuto de Autonomía catalán. Por otro lado, y a la vista de los acuerdos de 2004 sobre la posible participación de las regiones en las reuniones del Consejo como representantes de sus respectivos Estados miembros, parecen justificadas y necesarias las referencias a la participación autonómica en la fase ascendente de creación del Derecho comunitario. Finalmente, y en un tercer nivel, deberían incluirse algunas novedades previstas en la Constitución Europea, como las relativas a las funciones de los Parlamentos autonómicos en el control de la subsidiariedad o al papel de los Parlamentos nacionales respecto a las "cláusulas pasarelas" (art. 444).

Advirtió el Prof. Balaguer, sin embargo, de la gran incertidumbre que se cierne sobre el futuro de la Constitución Europea, consecuencia perversa de un proceso de ratificación excesivamente largo, resultado de su necesidad de legitimación. A la vista, precisamente, de sus deficiencias, coincidentes con las del proceso de reforma ordinaria, no sería extraño que se avanzara, según vaticinó, hacia mecanismos que prescindiesen del requisito de la unanimidad.

El segundo marco en el que se integra la reforma del EAA es el conformado por la CE y el resto del bloque de constitucionalidad. Una cronología lógica habría hecho preferible acometer la reforma constitucional antes de reformar los Estatutos de Autonomía. Sin embargo, y dado que no ha sido éste el itinerario seguido, se hace preciso analizar con detenimiento la compatibilidad entre las novedades que pretenden incluirse en las reformas estatutarias y la CE. A este respecto, no ofrecen problemas la introducción de catálogos de derechos sociales o el reconocimiento de instituciones y símbolos desarrollados al margen de las previsiones estatutarias; en idéntica posición se encontrarían los cambios en el sistema institucional propio de cada Comunidad Autónoma. Pero muy otra parece ser, sin embargo, la situación respecto a dos temas que parecían cerrados: el reparto competencial y el modelo de financiación.

Respecto al primero, todo parecía apuntar a que, una vez alcanzado el techo competencial máximo marcado por el límite del art. $149 \mathrm{CE}$, se trataba de un proceso acabado. No obstante, las aspiraciones de las CCAA que proponen en la actualidad la reforma de sus Estatutos, parecen decantarse por el uso, algo torticero, de la cláusula del art. 150.2 CE y acceder así a competencias en principio vedadas a ellas. En segundo lugar, a pesar del acuerdo sobre el mo- 
delo de financiación autonómica alcanzado en 2001, el correlato lógico a una ampliación competencial se encuentra en un aumento de los fondos disponibles por las CCAA.

La consecuencia de abrir a la modificación ambos ámbitos (competencias y financiación) vendría a suponer, sencillamente, la formulación de un nuevo modelo de Estado autonómico no susceptible, en apariencia, de culminar en momento alguno; en definitiva, una reforma constitucional encubierta.

B) La segunda ponencia, presentada por el Prof. Dr. D. Gregorio Cámara, también Catedrático de Derecho Constitucional de la Universidad de Granada, con el título "Principios y derechos en la reforma del Estatuto de Autonomía para Andalucía”, comenzó analizando el marco general en el que se está produciendo la reforma del EAA. En este sentido, y a diferencia de lo que parece estar ocurriendo en otras CCAA, todos los grupos políticos aceptan, formal y materialmente, los límites marcados por la Constitución. Dentro de este ámbito, las ideas de las que partiría la reforma serían tres: aumentar la calidad de las competencias, como reacción frente a los abusos, normalmente vía bases, cometidos por el Estado central; alcanzar un nivel competencial igual al del resto de comunidades históricas; y abordar las cuestiones simbólicas (v.g., la declaración de nación), sólo en la medida en que, de no hacerlo, pudiera implicar un menor nivel de autogobierno.

Este planteamiento ha llevado a que se adoptara una posición de cautela y prudencia, a la expectativa de la evolución de las demás reformas estatutarias en curso. Como primer resultado, el informe de la Ponencia para la reforma del EAA habría pasado de puntillas sobre las cuestiones competenciales, centrándose sus trabajos y alcanzando un amplio consenso en materia de principios y derechos.

Con los principios programáticos, muchos de ellos incluidos en el Preámbulo, al cual se prevé dar mayor extensión y contenido, se pretenden subrayar los aspectos más relevantes para la Comunidad andaluza. A juicio del Prof. Cámara, es en ellos donde el EAA, "norma directiva fundamental", se manifiesta más claramente en tanto que norma vinculadora de los poderes públicos, de ahí su especial importancia.

Por su parte, la mención y el reconocimiento expreso de un amplio abanico de derechos sociales supone ahondar en lo que el Prof. Cámara consideró un rasgo característico de la autonomía andaluza. En efecto, el EAA se caracterizaría por ser un Estatuto de Autonomía especialmente sensible al Esta- 
do social, por lo que parece conveniente profundizar en esta seña de identidad. En esta dirección parecen apuntar muchos de los objetivos cuya inserción se prevé en el nuevo título preliminar.

Estos principios y derechos, junto a los que se prevé la inclusión de unas políticas públicas que hagan realidad su contenido, encuentran, finalmente, su contrapartida en los deberes de los ciudadanos. Éstos, si bien pendientes de un desarrollo posterior, han sido ya esbozados en el informe de la ponencia, que señala como deberes de inexcusable cumplimiento el ejercicio de una ciudadanía solidaria y responsable, la contribución al sostenimiento del gasto público, la protección del medio ambiente y la participación en situaciones de emergencia.

C) En tercer y último lugar durante esta sesión, tuvo lugar la intervención del Prof. Dr. D. Miguel Revenga, Catedrático de Derecho Constitucional de la Universidad de Cádiz.

Como destacó desde el propio título de su ponencia, "El sistema de gobierno de la Comunidad Autónoma de Andalucía", sus reflexiones no se dirigieron tanto a las instituciones de gobierno como a la forma (sistema) de gobierno, aspecto éste tradicionalmente marginado de los debates políticos. De hecho, y a modo de punto de partida, comenzó por señalar que la realidad gubernativa con la que nos encontramos se caracteriza por la gran distancia que media entre el diseño institucional y la práctica política. De este modo, y a pesar de que el sistema de gobierno formalmente instaurado sea el modelo parlamentarista, las actuaciones de los agentes políticos tienden, por lo general, a realzar figuras distintas al Parlamento (v.g, los partidos políticos o sus líderes, único punto de referencia de los electores al elegir a sus representantes).

Esta situación, que explicaría, por ejemplo, la divergencia casi absoluta entre el sistema español y el inglés, aun siendo los dos formas de parlamentarismo, se debe a que la forma de gobierno no dependería tanto de la estructura institucional establecida en la Constitución y los Estatutos como de una actitud, de un talante o de una práctica política dadas. En última instancia, la vitalidad de la práctica parlamentaria descansaría sobre el necesario compromiso y la disposición, por parte de las mayorías, a asegurar la participación de las minorías.

Descendiendo al ámbito autonómico, se constata, en primer lugar, que ni la CE ni los actuales Estatutos de Autonomía contienen previsiones respecto a la forma de gobierno establecida para las CCAA. La Constitución se limita a 
establecer y regular sumariamente las principales instituciones de algunas Comunidades Autónomas (Parlamento, Consejo de Gobierno y TSJ). Los Estatutos de todas las CCAA, a falta de más indicaciones, adoptaron tales instituciones y completaron el resto de su regulación copiando el modelo parlamentario del Estado. Ante esta situación, los procesos de reformas estatutarias constituyen el momento para abordar esta cuestión, pero no parece, sin embargo, que vaya a ocurrir así. En efecto, los debates desarrollados en relación con las reformas estatutarias en marcha, parecen centrarse, esencialmente, en un posible aumento de las competencias de las CCAA, situación propia de las posturas políticas nacionalistas y, por emulación, de las demás fuerzas políticas autonómicas.

El caso andaluz sería la única, aunque parcial, excepción: el documento de bases para la reforma del EAA, de diciembre de 2003, estableció la "profundización democrática" como uno de los objetivos de la reforma; esta profundización, sin embargo, parece asociarse casi exclusivamente en los trabajos posteriores de la ponencia para la reforma del EAA a la paridad y a la transversalidad de género.

El Prof. Revenga lamentó, para el específico caso andaluz, que se estuviera dejando pasar la oportunidad de incluir los ajustes necesarios que eviten las discrepancias entre el sistema diseñado y la práctica política. Especialmente cuando, vía legislativa, ya se habían dado pasos en dicha dirección. En este sentido, estimó que entre las reformas relacionadas con la forma de gobierno ya producidas en Andalucía cabía destacar, especialmente, dos de ellas. En primer lugar, la efectuada en el Reglamento del Parlamento, que invirtió la grotesca regla conforme a la cual el grupo que podía dirigir más preguntas a los miembros del Consejo de Gobierno fuera el que aglutinara más diputados (lo que, previsiblemente, implicaba la pertenencia de ambos al mismo partido). En segundo lugar, la imposición de la paridad obligatoria en las listas electorales.

Quedaría, sin embargo, un nutrido grupo de cuestiones pendientes, relacionadas con la forma de gobierno en Andalucía y especialmente vinculadas al Derecho parlamentario: así, la inestable situación que se crea con la investidura automática del candidato de la lista más votada en caso de que transcurran dos meses sin que ningún candidato haya alcanzado la mayoría necesaria para ser Presidente de la Junta; la falta de reconocimiento al Presidente de la Junta del derecho de disolución de la Cámara, sólo previsto en la Ley de Gobierno, y la correlativa limitación que impida hacer coincidir las elecciones autonómicas con las nacionales; o la posibilidad de limitar el mandato del Presidente de la Junta. 
Durante el coloquio que siguió a su intervención, el mismo Prof. Revenga indicó la posibilidad de sumar otras medidas, no estrictamente de Derecho parlamentario, a las ya expuestas. Entre ellas estaría el deber de los candidatos a la presidencia de la Junta de Andalucía de mantener debates televisivos, de tal modo que éstos no fueran una concesión, sino una obligación. En esta misma línea, el Prof. Acosta Sánchez llamó la atención sobre la posible conveniencia de establecer la incompatibilidad del cargo de Presidente de la Junta de Andalucía con el de Presidente de un partido político de ámbito nacional.

V. A) En la mañana del jueves 21 de julio, las intervenciones empezaron con la ponencia del Prof. Dr. D. Manuel José Terol Becerra, Catedrático de Derecho Constitucional de la Universidad Pablo de Olavide de Sevilla, titulada "La reforma Competencial del Estatuto de Autonomía andaluz".

El Prof. Terol Becerra quiso comenzar destacando una característica de la Constitución de 1978: su proclive actitud hacia la descentralización política. En este sentido recordó que la Constitución había permitido a "las provincias con características históricas, culturales y económicas comunes", a "los territorios insulares" y a "las provincias con entidad regional histórica", acceder a su autogobierno y constituirse en Comunidades Autónomas. En opinión del Catedrático de la Pablo de Olavide resulta incuestionable que el pacto constitucional ha funcionado y que esta circunstancia permite esperar en el futuro que este pacto siga dando los mismos buenos frutos. De esta forma, puede considerarse inconcebible la construcción del Estado Autonómico sin el concurso complementario de los Estatutos de Autonomía -pese a la dificultad de su reforma-, imprescindibles para la actualización diacrónica de ese pacto constitucional.

Atendiendo al caso específico de su propia Comunidad Autónoma, el Prof. Terol Becerra estimó la oportunidad de una reforma del EAA, considerando que, lejos de buscar ninguna reforma del título VIII de la Constitución, lo que debe perseguirse es tan sólo la del EAA. Esta revisión estatutaria no se propone porque se juzgue inservible o ineficaz el Estatuto, sino porque, estimando todo lo contrario, se pretende enriquecerlo con nuevas aportaciones atentas a la cambiante realidad que impone el paso del tiempo.

Así, la experiencia de autogobierno acumulada por la Comunidad Autónoma de Andalucía desde la aprobación del EAA (ocurrida con la adopción de la Ley Orgánica 6/1981, de 30 de diciembre), habría permitido a los agentes políticos inmediatamente implicados en el ejercicio del autogobierno, a los agentes sociales y, en general, a la sociedad andaluza atenta a los avatares de 
la vida política, identificar con más precisión las políticas de mayor interés para la Comunidad Autónoma y los instrumentos más adecuados para su realización.

Según el Prof. Terol Becerra, la eficacia mostrada al efecto durante estos años por el EAA aconsejaría adoptar como punto de partida para el futuro su versión vigente que, en el terreno competencial, desarrolla la Constitución tan fielmente que ambas figuras normativas son representables gráficamente como las de un molde y de su vaciado. En ese sentido, la más legítima aspiración en la reforma del EAA debe consistir en seguir creciendo en el autogobierno, esto es, concentrar los esfuerzos en aspectos del mismo poco transitados todavía.

Ello obligaría, en opinión del Prof. Terol Becerra, a matizar la actual definición estatutaria de competencias. Unas veces, expresando con toda la precisión posible qué materias de las enumeradas en el artículo 149.1 de la Constitución merecen desgajarse de allí e incorporarse al Estatuto para incluirlas dentro de las potestades públicas, exclusivas o compartidas, de legislación y ejecución, que ostenta la Comunidad Autónoma de Andalucía. En otras ocasiones, aquéllas que coinciden con el ejercicio de potestades públicas en ámbitos materiales más propensos a los cambios, definiendo de un modo más amplio las materias competenciales asumibles por la Comunidad Autónoma de Andalucía.

B) La segunda ponencia del jueves fue a cargo del Prof. Dr. D. Javier Pérez Royo, Catedrático de Derecho Constitucional de la Universidad de Sevilla. La intervención tuvo como título "25 años de Estado Autonómico en España: un balance positivo" y empezó con una reflexión sobre el valor del término "Nación" en la Constitución en relación al proceso de reforma del Estatuto de Autonomía en Cataluña.

Según el Prof. Pérez Royo, en la Constitución Española esta palabra no actúa como límite del derecho a la autonomía, ni se proyecta normativamente sobre él en ningún lugar de la regulación que de su ejercicio se contiene en el título VIII CE. El término Nación figuraría en la Constitución con una dimensión simbólica más que con un valor normativo. El único valor que cabe atribuirle es el que deriva del adjetivo "nacional" como calificativo del sustantivo "soberanía", que se hace residenciar de manera exclusiva y excluyente en el "pueblo español" en el art. 1.2 CE. De esa forma, en el término "Nación" hay una vocación estatal y una afirmación directa, pero inequívoca, del principio de unidad política del Estado, que sí figura de manera expresa como lí- 
mite para el ejercicio del derecho a la autonomía a lo largo de todo el título VIII. Lo que la Constitución no tolera es que el ejercicio del derecho a la autonomía pueda convertirse en soberanía, fracturándose de esta manera la unidad del poder constituyente que reside en el pueblo español.

El Prof. Pérez Royo pasó entonces a analizar el Proyecto de reforma del Estatuto de Guernica que aprobó el Parlamento vasco en diciembre de 2003, deteniéndose sobre el hecho no de la afirmación de una nación vasca, sino de la afirmación de un "pueblo vasco" como titular de un poder constituyente originario diferenciado del poder constituyente del pueblo español. Esto hacía del mismo proyecto un quid inconstitucional. De esa forma, para el Catedrático de Sevilla, la reforma del Estatuto vasco no era independentista en sus resultados, sino en sus fundamentos, ya que suponía la ruptura del principio de unidad política del Estado.

Según el Prof. Pérez Royo nada parecido figuraría en el Proyecto de Reforma del Estatuto de Autonomía para Cataluña, ya que ahí el término "nación" carece de cualquier proyección en el articulado de la que pudiera derivarse, directa o indirectamente, una ruptura del principio de unidad política del Estado. Así, la diferencia fundamental en el uso de la palabra "nación" consistiría en que en la Carta Magna es símbolo de estatalidad, de soberanía, mientras que en el contexto de reforma del Estatuto solo sería portador de autonomía.

A partir de estas reflexiones la atención se trasladó a otros aspectos de la cuestión y particularmente al papel de los nacionalistas en los procesos de reformas. A su juicio, los nacionalistas son socios de gobierno incómodos, pero indispensables, porque aún cuando jurídicamente PP y PSOE podrían imponer su propio modelo, habría que atender a la "legitimidad" política. Ésta pasaría por contar con el apoyo de los nacionalistas, dado que éstos ostentan un elevado peso específico, e incluso mayoritario, en algunos parlamentos autonómicos, aunque no tengan representación en el Parlamento Español.

Finalmente, y corroborando las afirmaciones de los ponentes que le habían precedido los días anteriores al cuestionar el proceso abierto, el Prof. Pérez Royo admitió que lo lógico habría sido renovar la Constitución en primer lugar, para después abordar los cambios en los ámbitos autonómicos, siendo la especial coyuntura política la que ha obligado a hacer las cosas como están ocurriendo y sin que haya otras alternativas.

C) La última ponencia del día jueves 21 fue a cargo del Prof. Dr. D. Juan Cano Bueso, Catedrático de Derecho Constitucional de la Universidad de Al- 
mería y Presidente del Consejo Consultivo de Andalucía. Su intervención, que tuvo como título "¿Cómo y qué se quiere reformar del Estatuto de Autonomía para Andalucía?”, se desarrolló, de forma clara y concisa, alrededor de las principales preguntas que aún hoy guían la discusión sobre el estatuto autonómico andaluz: la necesidad real de la reforma, la oportunidad de realizarla en estos momentos, cómo realizarla y, sobre todo, con qué contenidos.

La ponencia, así, no pudo más que empezar con la pregunta que, con razón, podríamos definir como el leit motiv de todo el curso: ¿es verdaderamente necesaria una reforma estatutaria en Andalucía? El Prof. Cano Bueso quiso responder a ese "dilema" partiendo de un análisis sobre la redacción del actual EAA, que, en su opinión, fue realizado en un ambiente de inseguridad política y pactado desde la desconfianza. Así se explicaría, por ejemplo, la regulación de la designación del Presidente autonómico, que el Prof. Cano Bueso, considera inconstitucional tal y como está contemplada hoy en el EAA.

Según el Presidente del Consejo Consultivo de Andalucía, desde el momento de la aprobación del actual Estatuto, muchos e importantes cambios se han producido, tanto en el ámbito político como en el contexto social (como, sólo citando algunos, la consolidación de un sistema políticamente federal, la integración en la UE y, sin duda, la necesidad de dar una respuesta pública a los retos de la globalización), lo que habría inevitablemente producido el efecto de dejar desfasados algunos aspectos del Estatuto. Todo ello fundamentaría la necesidad de adaptar el EAA a la realidad actual dentro de la lealtad a la Constitución de 1978.

Respecto a cuándo debería emprenderse un proceso de reforma, el Prof. Cano Bueso estimó que la reforma del EAA está indisolublemente ligada a lo que debe ser la paralela reforma de la Constitución Española. Esta necesidad vendría marcada por las consecuencias de una eventual reforma del Senado, de la participación en las relaciones internacionales, y del sistema general de cooperación. Por ello mismo, el mejor modo de proceder a la reforma pasa por la necesidad, absolutamente imprescindible, de alcanzar un amplio consenso en torno a los grandes temas susceptibles de reforma. Estos vendrían a identificarse con las bases para la reforma elaboradas por el Ejecutivo andaluz.

Finalmente, respecto al contenido que debería tener la reforma, según el Prof. Cano Bueso es imprescindible establecer un control de los senadores por el Parlamento, proceder a una configuración de la Cámara de Cuentas y del Consejo Consultivo, a la regulación y control de los medios de comunicación públicos, así como a la protección de los derechos sociales adicionales, 
como los de la mujer, a fin de asegurar el preciado objetivo de avanzar hacia una democracia directa y más participativa. La modernización a nivel ecológico, democrático, institucional y respecto a la organización territorial y la financiación, la gestión de la Administración de Justicia, la necesidad de evitar el vaciamiento de competencias mediante la delimitación de materias competenciales, así como la duplicación administrativa en la organización territorial y los conflictos competenciales, representarían los demás objetivos que, en opinión del ponente, la reforma del EAA tendrá que plantearse.

VI. Finalmente tuvo lugar la sesión vespertina del mismo jueves 21 de julio, celebrada en la añeja Facultad de Derecho de la Universidad de Córdoba y con la que se cerraron estas Jornadas.

A) Fue el Prof. Miguel Agudo quien abrió esta última ronda de conferencias, enfrentando directamente un tema que, a modo de Guadiana, ya había hecho breves apariciones en casi todas las ponencias precedentes y que aún ocuparía buena parte de los debates de la tarde. En concreto, se trataba de la controversia sobre la posibilidad y oportunidad de incluir un catálogo de derechos sociales en el EAA.

A través de una esquema expositivo excepcionalmente claro, el Prof. Agudo planteó el acercamiento a la cuestión marcando, en primer lugar, los límites políticos del debate. Así, todo posicionamiento en la materia debería partir del reconocimiento del éxito del Estado de las Autonomías como la primera construcción jurídica, social y constitucional, capaz de reconocer la diversidad y la pluralidad y garantizar la unidad del Estado.

Desde esta perspectiva, y desde el entendimiento de que toda construcción política sólo queda justificada en la medida en que contribuye al aumento del bienestar social, los Estatutos de Autonomía aparecerían como instrumentos idóneos para concretar, normativamente, los compromisos políticos adoptados frente a las necesidades concretas de cada comunidad. A ello se sumaría, además, el especial valor normativo de los derechos en nuestro ordenamiento. En efecto, según denunció el Prof. Agudo, la discusión sobre la inclusión de una carta de derechos sociales queda viciada desde el momento en que se olvida el doble valor de los derechos fundamentales en las sociedades democráticas avanzadas: de un lado, y desde el plano subjetivo, como garantes de la libertad individual; de otro lado, en el plano objetivo, como elementos del ordenamiento jurídico y cúspide del sistema axiológico que informa todos los sectores del Derecho. Con estas premisas, quedarían suficientemente justificadas la oportunidad y la necesidad de la inclusión de un nuevo título en el 
EAA relativo a Derechos sociales y políticas públicas. No sólo por cuanto supondría un reconocimiento de derechos subjetivos (muchos de ellos ya existentes en la legislación ordinaria), sino, muy especialmente, en tanto que los consagraría como principios informadores de todo el ordenamiento jurídico andaluz.

Ahora bien, la traducción de este desiderátum político en términos jurídicos ha llevado a la doctrina a discutir sobre la posibilidad de que los Estatutos de Autonomía reconozcan y regulen derechos y deberes de los ciudadanos. Respecto de esta cuestión, autores tan autorizados como el Prof. Pérez Royo contemplan esta inclusión como una acción inconstitucional o inútil, señalando en abono de sus tesis la ausencia de un apoderamiento expreso en la Constitución para que las CCAA puedan crear un catálogo propio de derechos y deberes. El Prof. Agudo, sin embargo, consideró que debe partirse de tres datos constitucionales insoslayables: en primer lugar, que la Constitución no impide expresamente que los Estatutos incluyan una Carta de Derechos propia; en segundo lugar, que los contenidos que el art. 147.2 CE señala como contenido necesario de los Estatutos son sólo los mínimos, por lo que éstos pueden regular otras materias; finalmente, que resulta recomendable que se incluya la mencionada tabla como expresión del pluralismo político que, como valor superior del ordenamiento jurídico, afianza y preserva el principio democrático, vertebrador de nuestro Estado.

No existen, por tanto, argumentos constitucionales infranqueables para negar la posibilidad de incluir el catálogo de derechos. No obstante, el futuro catálogo sí encuentra una importante limitación respecto a los derechos que pueda regular en concreto. En efecto, del juego conjunto de los arts. 53 y 81 $\mathrm{CE}$, se desprende que la regulación de los derechos fundamentales y libertades públicas queda reservada al legislador orgánico y, por lo tanto, estatal. Esta limitación, además, vendría explicitada y reforzada por las cláusulas de los arts. 139.1 y 149.1.1 CE. El Prof. Agudo, sin embargo, recordó, en primer lugar, que el texto literal del art. 139.1 CE no impide regulaciones diferentes por las CCAA, sino trato discriminatorio a los españoles. Por su parte, que el Estado tenga competencia exclusiva sobre la regulación de las condiciones básicas que garanticen la igualdad de todos los españoles en el ejercicio de los derechos y en el cumplimiento de los deberes constitucionales (art. 149.1.1 $\mathrm{CE}$ ) impide que las CCAA puedan regular el contenido esencial de esos derechos, pero no excluye la posibilidad de regular determinados aspectos de los mismos en virtud de los títulos competenciales atribuidos. Por lo tanto, el EAA podría incluir concreciones de los derechos fundamentales en la medida en que no formasen parte del contenido esencial del derecho y ostentase com- 
petencias sobre la materia, así como aquellos derechos no contemplados en la Constitución y cuyo establecimiento pudiera derivarse también de un título competencial autonómico.

Junto a ellos, los derechos contenidos en la Sección Segunda del Capítulo Segundo, para los que sólo se prevé la reserva de ley (estatal o autonómica), también podrían ser objeto de su inclusión en el nuevo EAA. Finalmente, nada obstaría tampoco a que se pudieran transformar en derechos subjetivos los llamados "principios rectores" del Capítulo Tercero del Título Primero (arts. 39-52 CE).

B) La cuota femenina la cubrió la Prof ${ }^{\mathrm{a}}$ Dra. María Luisa Balaguer Callejón, Catedrática de Derecho Constitucional de la Universidad de Málaga y Consejera del Consejo Consultivo de Andalucía.

Su ponencia, "Reforma estatutaria y reforma de la Constitución", comenzó con la constatación de que, tras veinticinco años de vigencia, la CE no ha sido objeto de ninguna reforma verdaderamente sustancial. No obstante, frente a esta situación y en el marco político de las elecciones generales de 2004, el PSOE incluyó en su programa electoral una propuesta expresa de modificación constitucional. Dicha propuesta indicaba cuatro ámbitos cuya regulación resultaría, en la realidad actual, claramente insatisfactoria. En primer lugar, la estructura, composición y funcionamiento del Senado, teórica cámara de representación territorial (art. 69.1 CE) pero configurada, sin embargo, al margen del Estado autonómico. En segundo lugar, la incongruencia constitucional consagrada en el art. 57.1 CE al incluir, entre los criterios de sucesión a la Corona, la preferencia del varón sobre la mujer. En tercer lugar, la ausencia, cada vez más injustificada, de toda mención al proceso de integración europea en general y a la Unión Europea en particular, así como a las relaciones entre los ordenamientos jurídicos comunitario, estatal y autonómicos. Finalmente, la omisión, sólo justificable por causas históricas, de una mención expresa a cada una de las Comunidades y Ciudades Autónomas.

La dogmática constitucional ha calificado esta propuesta de reforma de insuficiente, por considerarla excesivamente reducida. No obstante, es en este contexto de tímidas modificaciones constitucionales en el que se inicia la reforma de los Estatutos. Por lo tanto, en opinión de la Prof ${ }^{\mathrm{a}}$ Balaguer cualquiera de las reformas estatutarias que se propongan, y que intente ser respetuosa con el actual marco constitucional, tendrá necesariamente un alcance muy limitado, dado que, expresado en símil espacial, el techo (la Constitución) se encuentra muy cerca de las cabezas de los Estatutos. 
Una vez expuesta la situación en que se encuentra el proceso de reforma constitucional, la Prof ${ }^{\underline{a}}$ Balaguer indicó las cuatro claves que permiten entender las reformas de los Estatutos de Autonomía. Tales claves, coincidentes con las principales innovaciones cuya inclusión se pretende, serían, en primer lugar, un aumento de las competencias, aspiración continua de las Comunidades históricas y, por emulación, de todas las demás. Como resultado del aumento de las competencias, se persigue, en segundo lugar, un nuevo modelo de financiación autonómica, de modo que ésta resulte suficiente para sufragar los gastos derivados del ejercicio de las nuevas competencias. La tercera innovación consistiría en la inclusión en los nuevos Estatutos de algunas figuras e instituciones surgidas en la práctica política autonómica (órganos de control externo, consejos consultivos) pero sin reconocimiento expreso en las actuales normas estatutarias. En cuarto lugar señaló, finalmente, la inclusión de Cartas de Derechos.

Todas estas modificaciones, y en especial la inclusión de Cartas de Derechos sociales, pueden contemplarse como mejoras sustanciales de los ordenamientos autonómicos. Ahora bien, sin perjuicio de esto, y a modo de conclusión, la Prof ${ }^{\underline{a}}$ Balaguer lamentó que las propuestas de reformas estatutarias no hayan incluido previsiones sobre otras materias, también necesitadas de revisión. De este modo, denunció cómo el actual sistema de fuentes de los ordenamientos autonómicos no prevé el recurso a la figura del Decreto-ley y, sólo en contados casos (v.g., Cataluña), al Decreto-legislativo.

En el posterior turno de preguntas, y al hilo de las reflexiones manifestadas por la Prof ${ }^{\mathrm{a}}$ Balaguer sobre la amplitud y el detalle al que podría descender el nuevo catálogo de Derechos sociales, el Prof. Agudo advirtió de la posible incompatibilidad entre Estado social y Estado democrático. En efecto, muchos, si no la totalidad, de los derechos que pasarían a incluirse en el EAA, existen en la actualidad como derechos de reconocimiento legal. En este sentido, al ser incluidos en la norma básica del ordenamiento andaluz y quedar, por tanto, fuera de la disposición del legislador ordinario, se estaría limitando el campo de acción y decisión de éste y, consecuentemente, las opciones por las que legítima y democráticamente podría decantarse.

C) Para finalizar, la ponencia con la que se clausuraron estas Jornadas corrió a cargo del Prof. Dr. D. Gerardo Ruiz-Rico, catedrático de Derecho Constitucional de la Universidad de Jaén, y se tituló "La reforma del Estatuto de Autonomía para Andalucía: conveniencia y alcance".

Su intervención dio comienzo con una reflexión sobre el esfuerzo por proyectar dentro del Estado de las Autonomías un sub-sistema político no sus- 
tancialmente distinto del modelo de gobierno previsto constitucionalmente para las instituciones políticas centrales. Según el Prof. Ruiz-Rico, el artículo 152 de la Constitución Española (CE) proporciona un esquema institucional básico, si bien circunscrito a las Comunidades de régimen especial. Por tanto, el diseño constitucional de una posible forma de gobierno autonómica debía haber quedado limitado a los pocos casos de las entidades que emplearon el artículo 151 (entre otras, Andalucía). Así, exclusivamente estas Comunidades -a través de sus correspondientes Estatutos de Autonomía- tenían que aplicar las previsiones estructurales impuestas por el artículo 152, es decir en primer lugar la necesaria creación de una Asamblea Legislativa, elegida por sufragio universal con arreglo a un sistema de representación proporcional, en segundo lugar, un Consejo de Gobierno, con funciones ejecutivas y administrativas, y dentro de aquél, un Presidente elegido por la Asamblea, entre sus miembros y, finalmente, la previsión de un Tribunal Superior de Justicia, donde culminaría la organización judicial en el ámbito territorial de la Comunidad Autónoma.

No obstante, durante el proceso autonómico se consensuó la necesidad de acomodar la estructura organizativa de todas las Comunidades, con independencia de la vía de acceso a la autonomía utilizada, a los perfiles diseñados por el artículo 152 de la Constitución. De esta forma el Prof. Ruiz-Rico quiso señalar que el modelo de gobierno proyectado por el constituyente tiene que responder -y tendrá que hacerlo en el futuro, mientras no se modifique el texto constitucional- a los dispositivos organizativos y relacionales de un sistema parlamentario.

Según el Catedrático de Jaén, las probabilidades que existen para modificar la forma de gobierno autonómica constitucionalizada son muy escasas. Paradójicamente, las CCAA "de vía rápida" (o del artículo 151) tienen en teoría un menor margen de disponibilidad sobre su propia forma de gobierno, si se compara con las Comunidades que accedieron por el procedimiento más lento del artículo 143. Las primeras, aun cuando representan a las Comunidades con un mayor grado de autoreferencialidad nacional, soportan una evidente "congelación" constitucional sobre su estructura político-institucional básica; no pueden modificarla sin que previamente se haya reformado el artículo 152 de la Norma fundamental.

Por el contrario, las CCAA de régimen ordinario cuentan, en principio y formalmente al menos, con una superior libertad estatutaria para implantar modelo "alternativos" de gobierno regional, al no estar condicionadas por ningún mandato constitucional. 
Con estas consideraciones el Prof. Ruiz-Rico terminó su ponencia, a la que siguió un breve momento dedicado a las preguntas y reflexiones por parte del público.

VII. Ni la canícula propia de las fechas y el lugar, ni el cansancio acumulado en el curso académico recién finalizado, deslucieron lo más mínimo el interés y el rigor de estas Jornadas. Todos los españoles, afectados o no por Estatutos en vía de reforma, asistimos en la actualidad a una nueva ocasión en que el Estado de las Autonomías parece enfrentar otra encrucijada. Así parecen testimoniarlo las declaraciones encontradas e inflamadas de partidos e instituciones, aventadas por unos medios de comunicación que, a veces, sólo aumentan la algarabía del foro. Por ello, el valor de estas Jornadas nos parece doble. De un lado, han constituido un lugar de diálogo sosegado, pero riguroso, sobre asuntos de primer orden de actualidad y relevancia. Por otro lado, han permitido que los asistentes conozcamos los juicios y aportaciones críticos de algunos de los principales juristas andaluces especializados en la materia.

Como ciudadanos, nos congratulamos sinceramente por la celebración de eventos que, como éste, nos hacen sentir parte activa de una sociedad democrática avanzada. Como asistentes, y especialmente como jóvenes investigadores iuspublicistas, nos sentimos obligados a agradecer la generosidad y cercanía de los ponentes y los organizadores, excepcionales investigadores y, como quedó demostrado, aún mejores docentes. 